Journal of Engineering and Applied Sciences 14 (16): 5880-5887, 2019

ISSN: 1816-949X

(C) Medwell Journals, 2019

\title{
Improvement of Security in Cloud of Health Care for the Human-Being via. Machine Learning
}

\author{
${ }^{1}$ Mohammed Layth Talal, ${ }^{2}$ Moayad Awny Sabri Alsamurai and ${ }^{3}$ Aymen Mudheher Badr \\ ${ }^{1}$ Department of Economics, College of Administration and Economic, \\ University of Diyala, Diyala, Iraq \\ ${ }^{2}$ General Directorate of Education of Diyala, Diyala, Iraq \\ ${ }^{3}$ Department of Political Science, College of Law and Political, University of Diyala,
}

Diyala, Iraq

\begin{abstract}
The requirement of the image processing advanced podium, since, it is regularly exorbitant includes financial expenditure and process periods. Accordingly, it is significant to approve cheap results to exchange conventional arrangements. Mention thoughts let to employ cloud computing to get big measure supplies for data processing. Accordingly, this method supplier's quick admission to on request of facilities with a large scale of accessibility. Thus with cloud facilities in its place of requests of inside home could certainly support establishments of health care for the human-being subcontract calculations to an outdoor gathering, thus, high reducing working expenditures. Nevertheless, the robust safety of data contrary to unreliable clouds and unlawful users is desired to avoid hateful data discovery. Now a days, several frameworks have been improved for allowing the users to supply and make operation to the same data via cloud computing. Broadly, there are several reasons to reinforcement via. systems of coding, distributing and occasionally a mixture of both them. Especially homomorphic coding systems, Facility-Oriented Architecture (FOA), Protected Multiparty Calculation (PMC) and Top-Secret Share systems (TSS) have a majority of the safety techniques for most of the entire present executions. The essential problem includes the operation of huge data analysis through the cloud via. mention methods is the calculation prices related to the mission of image processing. The primary and major challenge is to stop unlawful admission to medical registrations and special evidence of healthiness. In this study, a new method related to machine learning methods has been proposed to protect data processing in cloud surroundings. Naturally, the suggested work is to employ Artificial Neural Networks (ANNs) and Fuzzy $\mathrm{C}$-means Clustering ( $\mathrm{FCmC}$ ) to categorize pixels of the image within more proficiently. Moreover, an additional stage that has been combined known as the CloudSEC component, into the traditional structure of two-layered to decrease the danger of the possible discovery of medical evidence. Two sets of experiences have been achieved to estimate the suggested method. The simulated results prove that the employment of the ANNs is an effective idea for data safety and image division simultaneously. Actually, several hopeful results have been obtained which detect modern thoughts in order to elevate facilities of cloud in the scope of health care for the human-being.
\end{abstract}

Key words: Cloud computing, ANNs, FCmC, image processing, machine learning, PMC

\section{INTRODUCTION}

The prospect of outsourcing calculation processes can be provided by cloud computing, thus, permitting health care establishments to influence the benefits of cost. The employment of this idea allows customers to handle electronic health registers of patients remotely via. cloud computing based on imaging solutions. The main advantage of this idea is the capability to supplier widespread admission to requests of imaging without possessing to purchase and preserve of used materials. It is usually approved that cloud computing has entirely changed the method of health registers have been treated, kept, managed and employed. In this research, cloud supplier's provider an extensive diversity of Health Info Techniques (HITs) solutions to uphold schemes of electronic health schemes and happen the rising necessity for facilities of health care. Fascinatingly, the mushy exploitation of cloud requests, elastic sources administration, a pay-per-employment base sample for modification customers are several base features that describe facility of cloud (Hammid, 2013). This clearly

Corresponding Author: Mohammed Layth Talal, College of Administration and Economic, University of Diyala, Diyala, Iraq 
suggests that there would be a considerable development in a request for cloud acceptance because of its supplier's huge chances for health care establishments. Undesired a conventional prototype, the cloud employment takes security matters, since, data of customers have been usually registered and managed on centers of remote data. There is essentially a huge numeral of influences that may exactly make influence on solution of cloud deliberations, counting virtualization security dangers (Hammid, 2016), challenges of data registered position (Hammid et al., 2013), web technique of possibly unconfident registered (Marwan et al., 2016), missions of schemes interoperability (Hammid et al., 2016) and lawful restraints (Hammid et al., 2017). For example, homomorphic coding systems, Facility-Oriented Architecture (FOA), Protected Multiparty Calculation (PMC) and Top-secret Share Systems (TSS) have a majority of the safety techniques to confirm the lowest level of secrecy and confidentiality of health care registers for the patient. Nevertheless, in more examples, mention methods may not warrantee the necessities of Quality of Facility (QOF) which have been stated and well-known definite in the Facility Level Agreement (FLA). Firstly, numerical registers are very big that they have to be a time exhaustion in the procedure period. Secondly, mention techniques have to be inadequate with taking considered the medical data safety agreement. The essential objective of this study is to supplier health care establishments with a modest and effective cloud framework to investigate numerical registers via. just cloud sources. The structure shows how algorithms of machine learning can be employed to cope with safety problems in the field of data processing. Particularly, in this research, on Artificial Neural Networks (ANNs) dividers science they are getting a growing attention in multi-area division methods. Moreover, providing more protected processes, the suggestion is a respectable solution of distributed operation to observably develop the running time. The goal of the three stages construction is to grip supplementary cloud safety challenges and dangers, particularly secrecy and unlikability.

Literature review: Important secrecy worries might generally occur when storing out sourcing data and calculation to cloud suppliers. The secrecy of health care registers in storing of cloud typically includes encoding data of customers before communicating them into servers of off-site. In mention meaning, numerous methods are accessible to support contract with mention matter, containing AES, ECDH, RSA DES, 3DES, ECC. Unfortunately, mention approaches don't supplier the capability to accomplish protected data processing, since, they may not be employed in the encoded field. In this study, it's regarding mention challenge, the supplier and debate, methods for executing a protected cloud founded image processing. Challa et al. (2015) has been established a framework that employs typical of XML and Facility-Oriented Architecture (FOA) to shape systems of circulated software.

In this method, a block of numerous web facilities is shaped to procedure digital rejecters. Hence, every node is in charge of execution a definite mission. Precisely, the procedure of image investigation is primarily decayed into minor missions for every of which is charted to a separate cloud supplier. This technique is intended to confirm cooperation between commercial mechanisms throughout data processing. Nevertheless, secrecy safety is the essential difficulty of this method, since, its procedures just raw images. In the same term, image processing as a facility is established by Chiang et al. (2011). An APIs (Application Programming Interface) has been employed by Chiang et al. (2011) that mixes frontend facilities to investigate data of customers simply. Principally, FOA and image instruments are employed to facilitate the enhancement and placement procedures on the cloud podium. The suggestion is collected from numerous units to procedure data remotely. They contain a layer of presentation, a layer of facility, commercial logic and image instruments. The main worries that rise while using FOA technique in cloud computing is safety. Taking account into confidentiality safety, FOA is not considered to stop data expose both inside and outside. Unwanted the past approaches, confidentiality and safety thoughts are the important reasons beyond via. homomorphic encryption in data handling with cloud computing. More significantly, mention algorithms kinds uphold homomorphism for this reason one may make several arithmetic processes on encoded data. By Challa et al. (2015), the request protected cloud is enhanced for realizing positive outcomes by approving the encryption of homomorphic. The benefit has been taken for Learning With Error (LEWE) method, so as to handle digital register securely. In mention state, encode of customers in health data while cloud suppliers handle them on behalf of their clients without decoding. In specific, both of adding and multiplication are executed in an actual world request to assess the mentioned idea. Despite all benefits, the homomorphic method wasn't suitable for bigger capacities, particularly health images. Realistically, encryption of data via. mention method is exorbitant in computational, even for advanced processers, thus, may not be handled in applied imaging instruments. Also, (Gomathisankaran et al., 2013), suggested a framework which has outsourcing able of image processing. In 
mention scheme, cloud suppliers may investigate images without skimpy any evidence about plain text. Mention method may be simply realized with algorithms of homomorphic encryption to protect image processing. In this regard, the system of residue number is employed to encode data of clients before they have been moved to the cloud. Essentially, verge discovery over the filter of Sobel has executed to almost trial mention suggested framework. The essential weaknesses and boundaries of this mention technique are the time difficulty which blocks its exploitation in a cloud situation.

There is a fast-emergent necessity to progress a fast technique to manage a big amount of data. Accordingly, the dispersed data processing method was presented. Thus, an image is divided into numerous portions via. a Top-secret Share Scheme (TSS), agreeing to parallel processing. By Mohanty et al. (2012) there is an execution of the TSS algorithm for showing effective image investigation. Exactly, the suggested suppliers the capability to execute data imagining via. distant cloud facilities. In this situation, a mixture of TSS method and capacity version methods is exploited to uphold confidentiality and secrecy. Actually, this mention answer allows handling made small parts in their encoded arrangement. By Lathey and Atrey (2015) suggest an original technical process that allows on-line image processing by using cloud computing. The essential thought of the TSS method is to stop cloud suppliers to have admission to intimate data. Certainly, this mention answer is an instrument of encryption by using an inquiry has been transmitted to various nodes and every server will attempt to handle a portion of it. In doing so, may outsource image processing to servers of off-site without revealing the data of customers. Additional possible profits of the mention solution contain better dependability and execution. Obviously, the suggestion supplier's effective techniques to competently execute several image improvement processes like noise deletion, disparity and the verge of improvement. Thus, the suggestion will allow cloud computing facilities to be arranged in the field of health care. Normally, the essential challenge of using mention method is the request of the algorithm of image processing on a minor encoded section.

\section{MATERIALS AND METHODS}

Suggested frameworks: Clearly, the data safety scale supplied by off-site schemes may differ related to the selected cloud suppliers and Facility Level Agreement (FLA). Overall, the confidentiality matter turns into more complex with the cloud method, particularly when it turns into a field that is as critical as health care. According to these thoughts, robust privacy safety for medical registers is immediately wanted to stop inside or outside sections from the success of the secret data. More significantly, cloud suppliers have the prospect to handle encoded data simply while protective secrecy. In this regard, an original procedure has been suggested that attempts to improve confidentiality worries that happen while accomplishment image investigation remotely via. cloud computing. Essentially, the suggestion of cloud framework contains two main components for professionally contract to safety problems, i.e., construction of system and secrecy safety method.

Proposed architecture: Mostly, the standard construction of cloud computing typically discovers data of clients to numerous and significant safety impendences. In this regard, the proposal of a three-scale construction to improve data safety in the cloud situation. The consideration of such a scheme to include three various elements, i.e., client, cloudsec and cloud suppliers. In this prototypical, the cloudsec element primary encodes overall data of health by using the HTTPS/SSL protocol to protect data transmission. Secondary, mention element employs division method to retain the safety of medical images. After encoding, the CloudSEC transmits the data of the customer to an outside cloud supplier to handle them safely. In this situation, mention element is in charge of safeguarding passable confidentiality and safety for data of customer throughout the operation of cloud sources. In this situation Fig. 1 illustrates an overall indication of the suggested solution to decrease safety matters in cloud rated to image processing.

Moreover, the restriction of precise admission needs to cloud facilities via. an established of principles that describes the groups of lawful clients and the consents allocated to every customer. Now, the cloudsec unit supplies a diversity of actions to permit cloud suppliers to handle health registers without revealing private data for patients. Specifically, this unit can obviously limit admission and notice waste of cloud sources based on a predefined safety strategy. To uphold numerous regulator situations and policies there is a wide variety of admission regulator units that stop unlawful admission and employment data for patients known as optional admission regulator, obligatory admission regulator, role-based admission regulator and attribute-based admission regulator (Younis et al., 2014; Hammid and Sulaiman, 2018). The last is a very influential, active and elastic unit, regularly employed in 

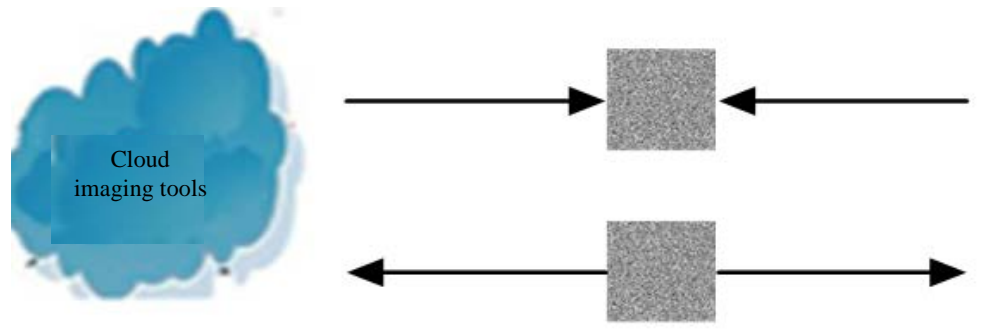

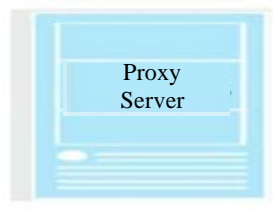

CloudSEC

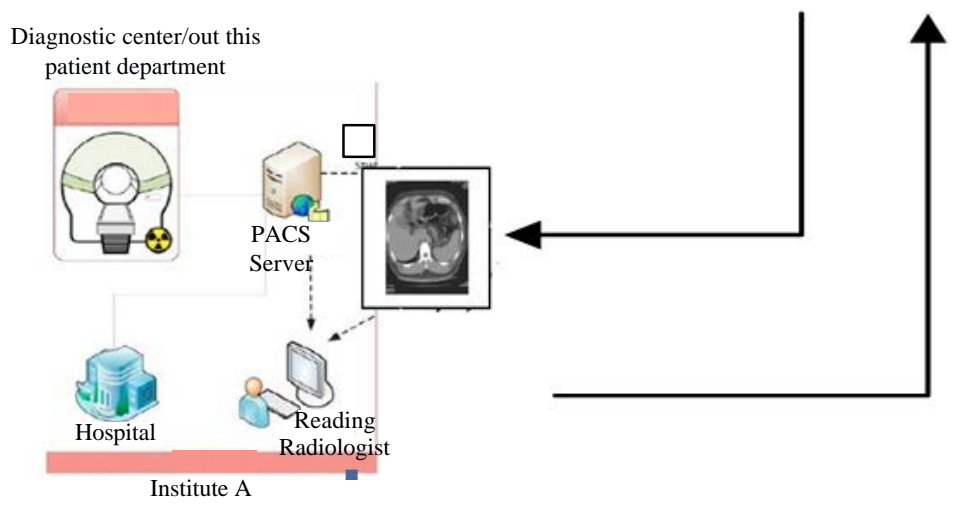

Fig. 1: An indication of cloud rated to image processing

the cloud to achieve safety necessities. In this study, the researchers mostly concentrate on data safety via. a division method, since, it is an easy and effective method. To realize this idea, a substitutional solution according to machine learning methods is employed to categorize data proficiently into dissimilar collections according to the image structures like the style, texture and colour.

Suggested data safety method: Algorithms of image division have been positively employed in a wide variety of requests but no one of them is instantly relevant in safety of data. The main objective of this study contains in executing this method to report safety matters in cloud facilities. The thought is to create pixels clusters with comparable structures in this way it would be probable to handle every area distinctly. In this framework, the primary exploration the pixel scale colour of the inserted image to classify every pixel. Secondly, the employment of mention structures as inserts to the Artificial Neural Networks (ANNs) for classification target. The last essentially is a managed algorithm of learning for training data set from applied skills. To this purpose, the objective depends on Fuzzy C-means Clustering ( $\mathrm{FCmC}$ ) to train ANNs classifiers to make increasing the productivity of linear classification. The overall concept of the classification method according to ANNs method is shown in Fig. 2.

Employed approaches: In this study an original secrecy safety technique has been presented by ANNs and

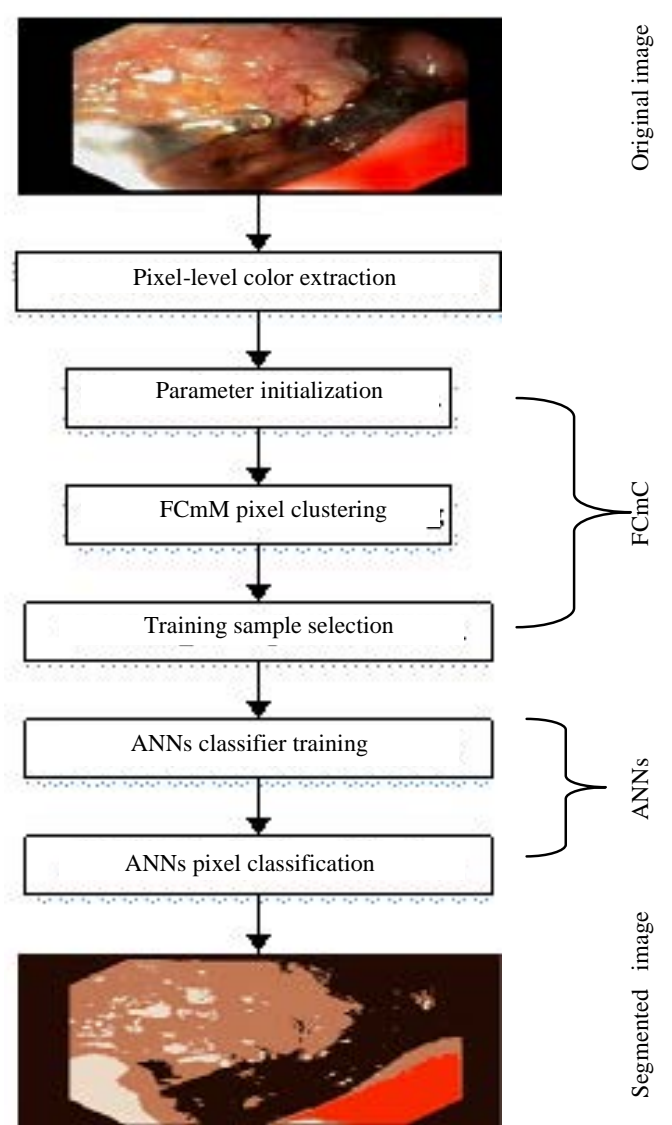

Fig. 2: The suggested technique for data safety 
$\mathrm{FCmC}$. The essential target is to make division an image into separate areas consisting pixels with scale image structures. Mostly, style, texture and colour structures are important parts employed to signify an image. Thus, the employment of FcmC in combination with ANNs is an actual method to stop the possible revelation of personal data. In this period, this technique is intended to realize rapid and correct outcomes. This section objects at supplying heavy vision into the suggested technique.

Fuzzy C-means Clustering (FCmC): The FCmM algorithm (Marwan et al., 2018) is a competent method for data investigation and unverified data classification. In disparity to conventional dividing approaches, every pixel may be fit to an additional one more cluster in fuzzy method related to its membership scores that is between 0 and 1 . The important contribution is to choose suitable centroids that minimize the variation function. This way may be simply realized by adjusting the membership matrix (X) arbitrarily as illustrated in Eq. 1:

$$
\sum_{\mathrm{i}=1}^{\mathrm{c}} \mathrm{X}_{\mathrm{ij}}=1, \forall \mathrm{j}=1, \ldots, \mathrm{n}
$$

Furthermore, mention algorithm employs the variation function, illustrated in Eq. 2, to data in classification:

$$
J\left(X, c_{1}, c_{2}, \ldots, c_{n}\right)=\sum_{i=1}^{c} J_{i}=\sum_{i=1}^{c} \sum_{j=1}^{n} X_{i j}^{m} d_{i j}^{2}
$$

Where:

$\mathrm{X}_{\mathrm{ij}} \quad=\quad$ Naturally between 1 and 0

$\mathrm{c}_{\mathrm{i}} \quad=$ Denotes to the cluster centroid of $\mathrm{i}$

$\mathrm{d}_{\mathrm{ij}}=$ Signifies the far of euclidian that between ith centroid $\left(\mathrm{c}_{\mathrm{i}}\right)$ and $\mathrm{jth}$ data point

$\mathrm{m} \in[1, \infty]=$ Signifies advocate of weighting

Artificial Neural Networks (ANNs): It's an easy and influential technique that mechanizes both classification and regression via. the concept of machine learning. characteristically, mention method employs widespread linear classifiers to assess data and distinguish patterns. finally, it trusts seriously on numerical learning concept settled by Vapnik (1995) to maximize analytical precision. Essentially, the selection of apply linear functions has been known as hypothesis, particularly in case of a high dimensional construction area. The main contribution of ANNs prototypical is to compute the hyperplane that greatest splits diverse clusters and maximize the minimum distance between any point of data (margin). In this sense, all points on the margin have been known as

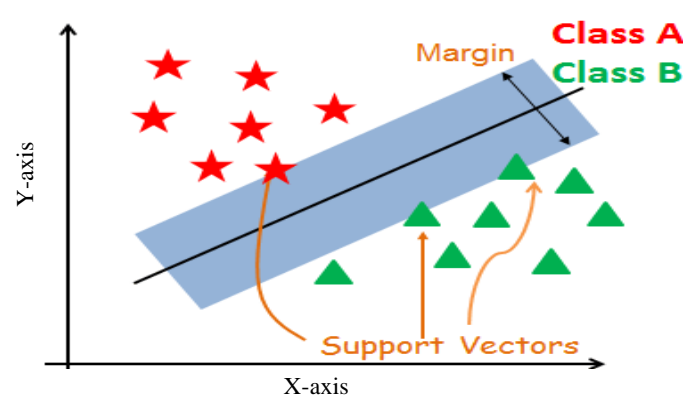

Fig. 3: Linear model of ANNs Model illustration

neurons. In a basic way, ANNs liner model has been signified which is the easiest style of this structure as shown in Fig. 3.

In mention structure, the employment is done in Eq. 3 to principally get the maximal probable margin of parting (Burges, 1998; Hammid and Sulaiman, 2018).

$$
\operatorname{marg} \text { in }=\arg \min d(x)=\arg \min \frac{\mid x \cdot w+b}{\sqrt{\sum_{i=1}^{d} w_{i}^{2}}}
$$

Where:

$\mathrm{w}=$ Indicates to choose hyperplane of standard vector

$\mathrm{x}_{\mathrm{i}}=$ The point of data $\mathrm{i}$

$\mathrm{y}_{\mathrm{i}}=$ Signifies a period of data point $\mathrm{i}(+1$ or -1$) \mathrm{NB}$ : not $1 / 0$

Classifier is computed according to the next expression: $f(x i)=\operatorname{sign}\left(w^{T} x_{i}+b\right)$. The practical margin of $X_{i}$ is illustrated by the next formula: yi $\left(w^{T} x_{i}+b\right)$. Scientifically language, hard-margin ANNs may be signified by difficulties of quadratic optimization which is specified by the next Eq. 4 (Vapnik, 1998; Kareem, 2018):

$$
\begin{aligned}
& \min \|\mathrm{f}\|_{\mathrm{k}}^{2}+\mathrm{C} \sum_{\mathrm{i}=1}^{\mathrm{i}} \xi_{\mathrm{i}} \\
& \mathrm{y}_{\mathrm{i}} \mathrm{f}\left(\mathrm{x}_{\mathrm{i}}\right) \geq 1-\xi \mathrm{i}, \text { for all } \mathrm{i}, \xi \geq 0
\end{aligned}
$$

As in the case of a dual problem, the ANNs can be expressed most succinctly as Eq. 5 for learning linear classifiers:

$$
\begin{aligned}
& \min \sum_{i=1}^{1} \alpha_{i}-\frac{1}{2} \sum_{i=1}^{1} \sum_{j=1}^{1} \alpha_{i} \alpha_{j} y_{i} K\left(x_{i}, x_{j}\right) \\
& 0 \leq \alpha_{i} \leq \mathrm{C} \text {, for all } \mathrm{i}
\end{aligned}
$$

where, $\zeta_{\mathrm{i}}$ amount signifies loose variables, preferably to be employed as the dimension error at point $\left(\mathrm{X}_{\mathrm{i}}, \mathrm{Y}_{\mathrm{i}}\right)$. In certainty, traditional methods like hard-margin don't 
typically consider the noise and doubt in the data. In order to report this matter, the software for margin ANNs employs naturally the idea of loose to prosses noisy of data. In this situation, ANNs expression turns into $y_{i}$ $\left(w^{\prime} x+b\right)>1-S_{k}$ where, $S_{k}$ is the permissible distance that splits data with the hyperplane without heretical the pre-clear restraints. According to mention thoughts, the employment of lagrangian variable is done to signify software margin ANNs as illustrated in Eq. 6 (Hammid etal., 2018a, b; Hammid and Sulaiman, 2017a, b):

$$
\begin{aligned}
& \min L=\frac{1}{2} \mathrm{w}^{\prime} \mathrm{w}-\sum \lambda_{\mathrm{k}}\left(\mathrm{y}_{\mathrm{k}}\left(\mathrm{w}^{\prime} \mathrm{x}_{\mathrm{k}}+\mathrm{b}\right)+\mathrm{S}_{\mathrm{k}}-1\right)+\alpha \sum \mathrm{s}_{\mathrm{k}} \\
& 0 \leq \alpha_{\mathrm{i}} \leq \mathrm{C} \text {, for all } \alpha_{\mathrm{i}}
\end{aligned}
$$

\section{RESULTS AND DISCUSSION}

Clearly, the division is a competent technique to generate homogeneous portions based on the chosen structures. To realize this purpose in a short and easy way, the employment of colour scale to classify every pixel of the image to realize pixel according to the division. In order to execute the suggested technique, the contribution depends on the Libanns (Chang and Lin, 2001) to simply construct ANNs. Actually, Libanns is a competent and influential open exporter library machine learning instrument that achieves classification for data. In specific, the chosen the one-against-one method in the multiple period ANNs execution. In this period, the execution of the algorithm of FCmC is done to train ANNs classifiers via. colour structure. To prove the precision of the suggested division technique, the applying of this method is done on RGB colour image in JPG setup. In this situation, the division of the secret image is excited into four areas based on colour of the pixel. In this thought, the employment of the division method is executed to get a numerous fundamental image processing in every area. Following, the combination of these portions is handled to contract the last image. In this way, the accomplishment of two fundamental purposes, i.e., image improvement and filter of Gaussian (Fig. 4a).
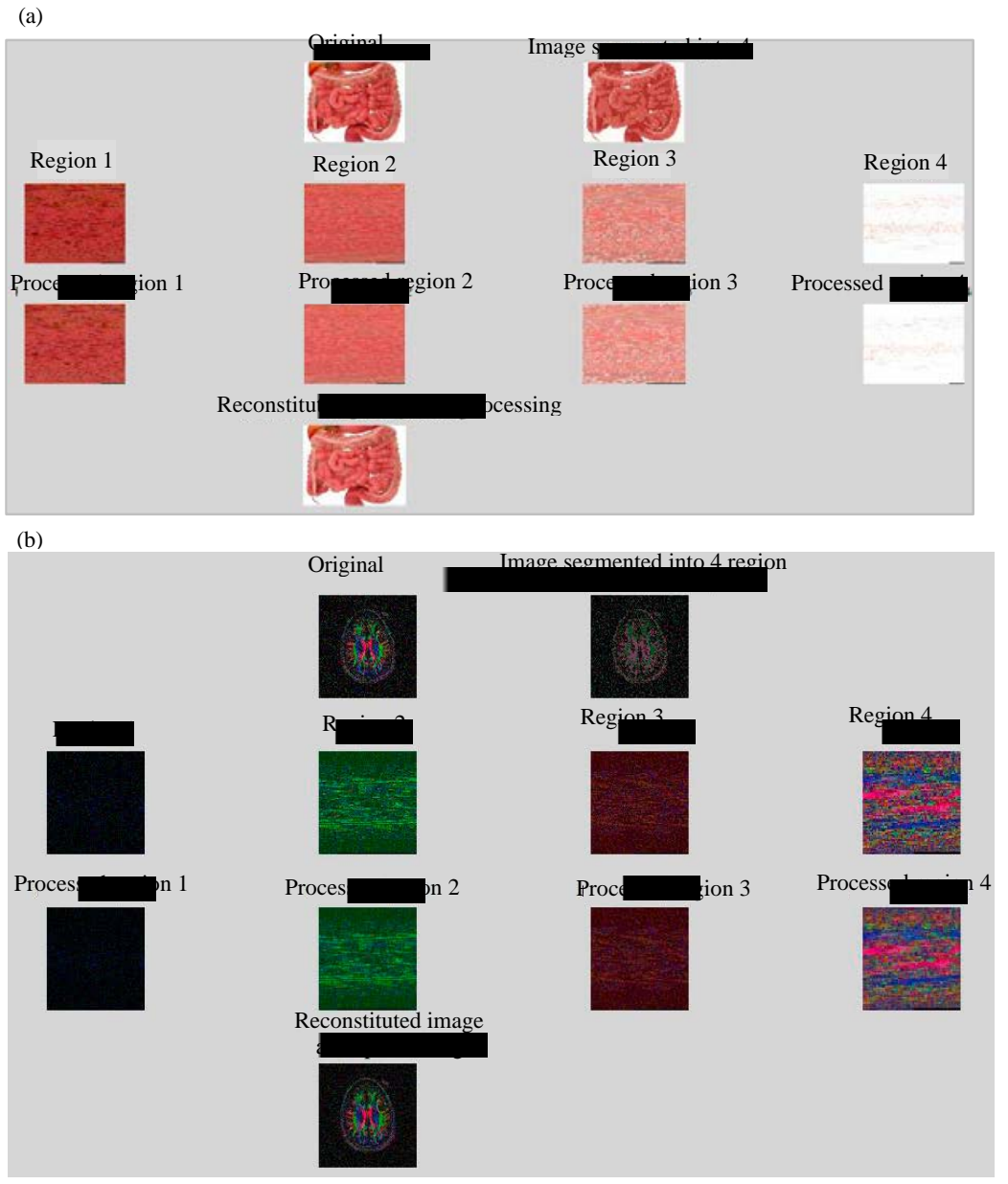

Fig. 4: Image processing using ANNs-FCmC Model; a) Gaussian filtering and b) Pixel improvement 
Firstly, the applying of Gaussian filtering to hazy images and get rid of noise and thereby developing the visual value of the handled image. In this setting, the pixels of the image are gathered into 4 slight areas. Then, the applying Gaussian filtering is executed on every portion. following, the reorganize these handled areas to reach the ultimate the results. Secondly, the changing of the pixel value is satisfied to improve image quality. In order to assess the accuracy of the suggested method, the tests are shown on two RGB images as illustrated in Fig. 4b.

\section{CONCLUSION}

Gratitude to cloud computing, Software as a Facility (SAF) prototype is an effective solution to simplify the enhancement of accessible Health Information Techniques (HITs). Nevertheless, the safety matter is the main importance of medical image investigation through cloud computing. The literature of review outcomes displays that present articles have boundaries concerning safety and secrecy. In this regard, the employment of hybrid method based on two algorithms of ANN and $\mathrm{FCmC}$ to be divided an image into several portions depending on the colour of pixels. The primary concept beyond this thought is that the cloud delivers might not expose mechanically secreted medical data. The numerical results prove the advantage of the machine learning mixture and division to enhance the security of the data. Therefore, the suggested technique provides the prospect of processing RGB bright images using cloud sources in a protected method.

More significantly, the application of two approaches for image improvement, i.e., pixel density and filtering of Gaussian. The proposed future work will concentrate on employed on more multifaceted image processing processes. Furthermore, the insinuation of employment characteristic of pixel quality as a supplementary parameter to improve image division. Also, the employment of identity prototype instead of colour pixel which contains two elements, i.e., the filter of Gabor and window of the local image. In the same term, the employment of datasets of the strong medical image with suitable data size, footnote, reality and reusability. This method can have an important capacity to correctly train data and excerpt the optimum advantage. From this viewpoint, this method would make benefit cloud delivers to simply defend data secrecy, averting exorbitant encryption approaches.

\section{ACKNOWLEDGEMENT}

We would like to thank Department of Computer, College of Engineering, University of Diyala, for assistance and development our study for comments that greatly improved the manuscript and we thank reviewers for their instructions and guidance.

\section{REFERENCES}

Burges, C.J.C., 1998. A tutorial on support vector machines for pattern recognition. Data Mining Knowl. Discov., 2: 121-167.

Challa, R., G. VijayaKumari and B. Sunny, 2015. Secure image processing using LWE based homomorphic encryption. Proceedings of the 2015 IEEE International Conference on Electrical, Computer and Communication Technologies (ICECCT), March 5-7, 2015, IEEE, Coimbatore, India, ISBN: 978-1-4799-6084-2, pp: 1-6.

Chang, C.C. and C.J. Lin, 2001. LIBSVM: A library for support vector machines. Software, 80: 604-611.

Chiang, W.C., H.H. Lin, T.S. Wu and C.F. Chen, 2011. Bulding a cloud service for medical image processing based on service-orient archtecture. Proceedings of the 2011 4th International Conference on Biomedical Engineering and Informatics (BMEI) Vol. 3, October 15-17, 2011, EEE, Shanghai, China, ISBN:978-1-4244-9351-7, pp: 1459-1465.

Gomathisankaran, M., X. Yuan and P. Kamongi, 2013. Ensure privacy and security in the process of medical image analysis. Proceedings of the 2013 IEEE International Conference on Granular Computing (GrC), December 13-15, 2013, IEEE, Beijing, China, ISBN:978-1-4799-1282-7, pp: 120-125.

Hammid, A.T. and M.H. Bin Sulaiman, 2017b. Priority of Kaplan Turbine and small hydropower plants over other resources: An overview. J. Eng. Appl. Sci., 12: 8695-8705.

Hammid, A.T. and M.H. Sulaiman, 2017a. Optimal long-term hydro generation Scheduling of Small Hydropower Plant (SHP) using metaheuristic algorithm in Himreen lake dam. Proceedings of the MATEC Web Conference on UTP-UMP Symposium on Energy Systems (SES'17) Vol. 131, October 25, 2017, EDP Sciences, Les Ulis, France, pp: 04017-04021.

Hammid, A.T. and M.H.B. Sulaiman, 2018. Series division method based on PSO and FA to optimize long-term hydro generation scheduling. Sustainable Energy Technol. Assess., 29: 106-118.

Hammid, A.T., 2013. Applications of tuning control actions for the efficient load/frequency control in steam turbine. Intl. J. Curr. Eng. Technol., 3: 1895-1898. 
Hammid, A.T., 2016. Direct on line starter motor and reverse system in Allen-Bradley PLC. Diyala J. Pure Sci., 12: 132-148.

Hammid, A.T., A.K. Bhardwaj and S. Prakash, 2013. Design remote power control $\mathrm{I} / \mathrm{O}$ data acquisition system and control on home automation. Intl. J. Electron. Commun. Comput. Eng., 4: 528-535.

Hammid, A.T., M. Hojabri, M.H. Sulaiman, A.N. Abdalla and A.A. Kadhim, 2016. Load frequency control for hydropower plants using PID controller. J. Telecommun. Electron. Comput. Eng., 8: 47-51.

Hammid, A.T., M.H.B. Sulaiman and A.A. Kadhim, 2017. Optimum power production of Small Hydropower Plant (SHP) using Firefly Algorithm (FA) in Himreen Lake Dam (HLD), Eastern Iraq. Res. J. Appl. Sci., 12: 455-466.

Hammid, A.T., M.H.B. Sulaiman and A.N. Abdalla, 2018a. Prediction of small hydropower plant power production in Himreen Lake dam (HLD) using artificial neural network. Alexandria Eng. J., 57: 211-221.

Hammid, A.T., M.H.B. Sulaiman and O.I. Awad, 2018b. A robust firefly algorithm with backpropagation neural networks for solving hydrogeneration prediction. Electr. Eng., 100: 2617-2633.

Kareem, H.J., 2018. Control on hydropower plant using fuzzy neural network based on right-angle triangle membership. J. Adv. Res. Dyn. Control Syst., 10: 1239-1250.
Lathey, A. and P.K. Atrey, 2015. Image enhancement in encrypted domain over cloud. ACM. Trans. Multimedia Comput. Commun. Appl., 11: 1-24.

Marwan, M., A. Kartit and H. Ouahmane, 2016. A secure framework for medical image storage based on multi-cloud. Proceedings of the 2016 2nd International Conference on Cloud Computing Technologies and Applications (CloudTech), May 24-26, 2016, IEEE, Marrakech, Morocco, ISBN:978-1-4673-8895-5, pp: 88-94.

Marwan, M., A. Kartit and H. Ouahmane, 2018. Security enhancement in healthcare cloud using machine learning. Procedia Comput. Sci., 127: 388-397.

Mohanty, M., P. Atrey and W.T. Ooi, 2012. Secure cloud-based medical data visualization. Proceedings of the 20th ACM International Conference on Multimedia (MM '12), October 29- November 02, 2012, ACM, New York, USA., ISBN:978-1-4503-1089-5, pp: 1105-1108.

Vapnik, V., 1995. The Nature of Statistical Learning Theory. 2nd Edn., Springer, New York, USA., ISBN-13: 9780387945590 , Pages: 188.

Vapnik, V.N., 1998. Statistical Learning Theory. Vol. 3, John Wiley \& Sons, NewYork, USA., ISBN:978-0-471-03003-4, Pages: 740.

Younis, Y.A., K. Kifayat and M. Merabti, 2014. An access control model for cloud computing. J. Inf. Secur. Appl., 19: 45-60. 